\title{
Mobile Health and Telehealth Interventions to Increase Physical Activity in Adolescents with Obesity: a Promising Approach to Engaging a Hard-to-Reach Population
}

\author{
Mary Ellen Vajravelu ${ }^{1,2}$. Silva Arslanian ${ }^{1,2}$
}

Accepted: 19 August 2021 / Published online: 1 October 2021

(c) The Author(s), under exclusive licence to Springer Science+Business Media, LLC, part of Springer Nature 2021

\begin{abstract}
Purpose of Review Adolescence represents a critical time to set habits for long-term health, yet adequate rates of physical activity are uncommon in this age group. Mobile technology use, however, is ubiquitous. We review advantages and challenges posed by mobile health (mHealth) and telehealth-based physical activity interventions aimed at adolescents.

Recent Findings Mobile Health (mHealth) and telehealth interventions to increase physical activity in adolescents include use of wearable activity trackers, text messages or apps, and video visits with exercise specialists. Definitions and goals for physical activity differ across interventions, and methods of activity measurement also vary. User engagement is often poor, if tracked at all. No identified studies included use of behavioral economics-informed engagement strategies.

Summary Intervention designers must plan for ways to maximize engagement and to reliably measure the intended outcome. Although mHealth and telehealth interventions have advantages such as scalability and acceptability, potential pitfalls must be addressed before widespread implementation.
\end{abstract}

Keywords Physical activity $\cdot$ Adolescence $\cdot$ Mobile health $\cdot$ Telehealth $\cdot$ Text messaging $\cdot$ Obesity

\section{Introduction}

Despite some degree of heritability, human physical activity remains a potentially modifiable determinant of health and disease [1]. Both physical activity tendencies [2] and obesity [3] tend to track from childhood to adulthood, so adolescence represents a critical time to set habits for long-term health. Although adolescents should engage in 60 minutes or more of moderate-to-vigorous physical activity daily, as well as muscle- and bone-strengthening activities 3 days

This article is part of the Topical Collection on Etiology of Obesity

Mary Ellen Vajravelu

MaryEllen.Vajravelu@pitt.edu

1 Division of Pediatric Endocrinology, Diabetes, and Metabolism, UPMC Children's Hospital of Pittsburgh, University of Pittsburgh School of Medicine, 4401 Penn Ave, Faculty Pavilion, 6th Floor, PA 15224 Pittsburgh, USA

2 Center for Pediatric Research in Obesity and Metabolism, UPMC Children's Hospital of Pittsburgh, University of Pittsburgh School of Medicine, Pittsburgh, PA, USA per week, only approximately one-eighth to one-third of US high school students met these goals in 2011-2015 [4••]. In addition to its numerous benefits to physical health, including decreased fasting insulin, increased insulin sensitivity [5-7] and improved body composition [7], physical activity confers improvements in mental health, including depressive symptoms, stress, and quality of life [8]. With rising rates of obesity and mental health challenges, both of which appear to have been worsened by changes to daily life associated with the COVID-19 pandemic [9], effective and scalable interventions are needed to promote health behavior change targeting physical activity in adolescents. How to achieve this, however, has been a long-standing challenge.

One promising method to reach adolescents, both to inform and to encourage them to engage in physical activity, is through mobile technology, utilizing the smartphones to which $95 \%$ of adolescents currently have access [10]. Mobile health, or mHealth, refers to the use of mobile technology such as smartphone or tablet apps or text messages to engage end-users in improving their own health. Telehealth, a more general category, includes patient-clinician communication via video-conferencing or other methods of remote access to provide health assessment or counseling. 
Connected devices such as Bluetooth-enabled activity monitors can assist with physical activity monitoring and feedback. In-person lifestyle interventions [11] face challenges with scalability due to the need for dedicated space and multidisciplinary staff, and they have high attrition rates, particularly among economically disadvantaged and minority youth [12]. In light of these challenges, mHealth may be especially useful in the development of lifestyle interventions that are less resource-intensive and more engaging.

Mobile health strategies have been used successfully for a wide variety of health behavior change interventions in youth [13]. Despite their promise, however, telehealth and mHealth-based interventions may not succeed without careful consideration of the end-users and ways in which they may become and stay engaged. Use of behavior change theories [14] and concepts from behavioral economics may help to motivate and reinforce habit development. In this review, we discuss important considerations for the development of mHealth physical activity interventions for adolescents, including potential pitfalls and possible solutions.

\section{What Is the Goal? Defining “Physical Activity"}

An initial task when designing any physical activity-promoting intervention is to define what is meant by physical activity. As described by the 2018 Physical Activity Guidelines for Americans, both an activity's timing and intensity should be specified $[4 \bullet \bullet]$. For example, compared to a no-exercise control condition, aerobic exercise done before a high-fat meal reduces postprandial triglycerides in overweight adolescents [15]. While single sessions of moderate-to-vigorous physical activity can have same-day positive impacts on insulin resistance, blood pressure, anxiety symptoms, sleep, and cognition, many benefits such as improved fitness and disease risk reduction must

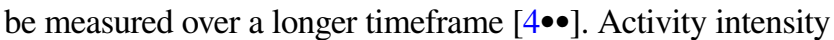
can be divided into light, moderate, and vigorous as defined by absolute measurements based on energy expenditure (multiples of the metabolic equivalent of task, MET, where 1 MET is the energy expenditure rate at rest). Alternatively, relative intensity can be used to take into account an individual's level of fitness: for example, on a 0 to 10 scale, with sitting rated as 0 and highest effort level as 10 , moderate-intensity activity would be rated as 5-6 and is associated with faster heart rates and faster breathing [4••]. For adolescents, typical moderate-intensity activities include brisk walking, bicycle riding, swimming, and house or yard work, while vigorous-intensity activities include running,

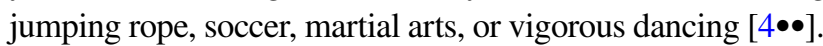

In addition to aerobic activities, per the 2018 Physical Activity Guidelines for Americans, adolescents should also engage in muscle- and bone-strengthening activities

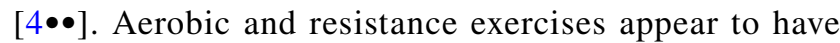
different physiological effects, which may also differ by sex. For example, in a randomized controlled trial by Lee et al. of aerobic versus resistance exercise in adolescent boys with obesity, both aerobic and resistance exercise were effective at reducing abdominal fat and intrahepatic lipid content, but only resistance exercise was associated with significant improvements in insulin sensitivity [16]. In contrast, in a separate randomized control trial by the same investigators of aerobic versus resistance exercise in adolescent girls with obesity, only aerobic exercise was effective at reducing visceral and liver fat and improving insulin sensitivity [7]. However, differences in response to resistance training could also have been related to lower uptake and engagement in resistance training by girls. In a third randomized controlled trial by the same investigators that included both adolescent boys and girls with obesity and evaluated aerobic versus resistance versus combined aerobic and resistance exercise, combined exercise was similar to aerobic exercise in the improvement in insulin sensitivity and reduced ectopic fat, while resistance exercise alone did not result in liver fat reduction [17]. Although beneficial in other ways as described above, neither aerobic nor resistance exercise, alone or in combination, altered resting metabolic rate in adolescents with overweight or obesity [18].

With physical inactivity described as the world's fourth highest risk factor for mortality [19], the American Heart Association recommends that adults "sit less, move more" [20]. In youth, however, the association between sedentary behavior and outcomes appears to depend heavily on context: for example, time spent reading or working on homework may not be detrimental to health, while TV viewing and screen time are more consistently associated with negative health outcomes [21]. This discrepancy may be due at least in part to the greater consumption of poorer-quality, energy-dense foods that tends to occur during TV watching [22]. Due in part to the challenge of accurately measuring sedentary time and its context, appropriate limits on screen time are difficult to determine $[21,23]$. Interventions targeting sedentary behavior should determine how it will be measured while considering potential drawbacks. Measurements done objectively via accelerometry should account for missing data, direct observation should acknowledge the inability to measure total sedentary time, and self-report measurements should be done with the caveat of potentially low reliability and validity due to recall and social desirability biases [23]. Explicit and carefully considered 
Table 1 Considerations when designing physical activity interventions for adolescents

\begin{tabular}{|c|c|c|c|c|}
\hline Goal & Goal details & Measurement & Outcomes & Example Interventions \\
\hline $\begin{array}{l}\text { Increase physical } \\
\text { activity }\end{array}$ & $\begin{array}{l}\text { - Frequency and timing } \\
\text { - Intensity (light, moderate, } \\
\text { vigorous), including relative } \\
\text { versus absolute } \\
\text { - Aerobic versus resistance } \\
\text { activities }\end{array}$ & $\begin{array}{l}\text { - Wearable activity } \\
\text { trackers } \\
\text { - Observation } \\
\text { - Self-report }\end{array}$ & $\begin{array}{l}\text { - Step count } \\
\text { - Time } \\
\text { spent in } \\
\text { activity } \\
\text { - Distance }\end{array}$ & $\begin{array}{l}\text { 1. Complete at least } 10 \text { min of moderate to } \\
\text { vigorous physical activity (e.g., brisk walking } \\
\text { or jogging) } 3 \text { times per week before dinner. } \\
\text { Participants wear commercial activity tracker } \\
\text { on wrist while awake. Both step count and time } \\
\text { spent in activity captured. } \\
\text { 2. Complete at least } 15 \text { min of resistance activities } \\
\text { twice weekly at time of participant's choosing. } \\
\text { Participants self-report to investigators using two- } \\
\text { way texting at start and completion of activity and } \\
\text { receive encouraging feedback. Total time spent } \\
\text { weekly and sessions per week captured. }\end{array}$ \\
\hline $\begin{array}{l}\text { Reduce sedentary } \\
\text { behavior }\end{array}$ & $\begin{array}{l}\text { - Context (e.g., screen time } \\
\text { versus homework) } \\
\text {-Pattern (e.g., interspersed } \\
\text { or uninterrupted) }\end{array}$ & $\begin{array}{l}\text { - Wearable activity } \\
\text { trackers } \\
\text { - Observation (not } \\
\text { for total daily } \\
\text { sedentary time } \\
\text { assessments) } \\
\text { - Self-report }\end{array}$ & - Time & $\begin{array}{l}\text { 1. Participants wear commercial activity trackers } \\
\text { while awake to obtain baseline total daily (non- } \\
\text { sleeping) time spent sedentary. Participants asked } \\
\text { to reduce screen time by } 30 \text { min daily from baseline } \\
\text { and provided examples of how to do so (e.g., place } \\
\text { smartphone out of sight during dinner or before } \\
\text { going to bed). Change in time spent in sedentary } \\
\text { behavior from baseline to intervention end assessed. } \\
\text { 2. Ecological momentary assessment (EMA) used } \\
\text { to collect participant self-report of activities } \\
\text { throughout the day, then mapped to sedentary } \\
\text { versus active behaviors. Participants coached } \\
\text { via an app with individualized notifications to } \\
\text { interrupt sedentary behaviors during times at } \\
\text { which EMA self-report identified the participant } \\
\text { to be most sedentary at baseline. Follow-up } \\
\text { EMA after the intervention assesses whether a } \\
\text { greater proportion of time was reported as non- } \\
\text { sedentary behavior. }\end{array}$ \\
\hline
\end{tabular}

measurement of a physical activity intervention's impact on sedentary behavior would add to the limited evidence base of the effect of sedentary behavior reduction on adolescent health. Table 1 highlights details that should be considered when designing adolescent-targeted interventions to increase physical activity and/or decrease sedentary behavior.

\section{How Will the Activity Be Measured? The Use of Wearable Physical Activity Trackers}

Objective measurements, rather than subjective measures such as a 3-day physical activity recall, are necessary for reliable assessments of physical activity and sedentary behavior in adolescents with overweight or obesity. As shown by Rockette-Wagner et al., adolescents with type 2 diabetes significantly underestimate time spent in sedentary behavior relative to moderate-to-vigorous physical activity when completing a 3-day physical activity recall (Fig. 1) [24]. In a study of children and adolescents with severe obesity, Lockwood et al. found similar discrepancies between self-report of physical activity and accelerometer-measured activity; they suggest that overestimation of self-reported vigorous activity may be due to increased perceived exertion during physical activity in youth with obesity [25]. Fortunately, wearable activity trackers, which use sensors such as accelerometers or altimeters to track movement, make objective measurement of physical activity straightforward and can provide real-time and summarized feedback for selfmonitoring of activity. There are a variety of commercially available devices or apps, both stand-alone and integrated into smartwatches or smartphones. For the purpose of this review, we will discuss only devices or apps that provide feedback to the wearer and are thus more applicable to scalable mHealth interventions.

Due in part to the constant introduction of new devices and brands, it is challenging to thoroughly validate consumer wrist-worn wearables prior to use in research [26]. In a study by Henriksen et al. assessing the availability of wrist-worn fitness wearables from 2011 to 2017 , the authors identified over 400 unique devices from 132 different brands [26]. They noted that the five brands most commonly used in research projects were Fitbit, Garmin, Misfit, Apple, and Polar, but that Fitbit was the most common wearable device registered in ClinicalTrial.gov studies by ten-fold. In a systematic review that included 67 studies evaluating the accuracy of 
Fitbit activity trackers used by adults in controlled and freeliving settings, Feehan et al. conclude that Fitbit devices met acceptable accuracy for step count approximately $50 \%$ of the time, underestimating in controlled testing and overestimating in free-living settings [27]. Fitbit devices also tended to overestimate time spent in higher-intensity activities. The validity of Fitbit devices or other consumer wrist-worn activity trackers has been under-evaluated in children and

\section{A}

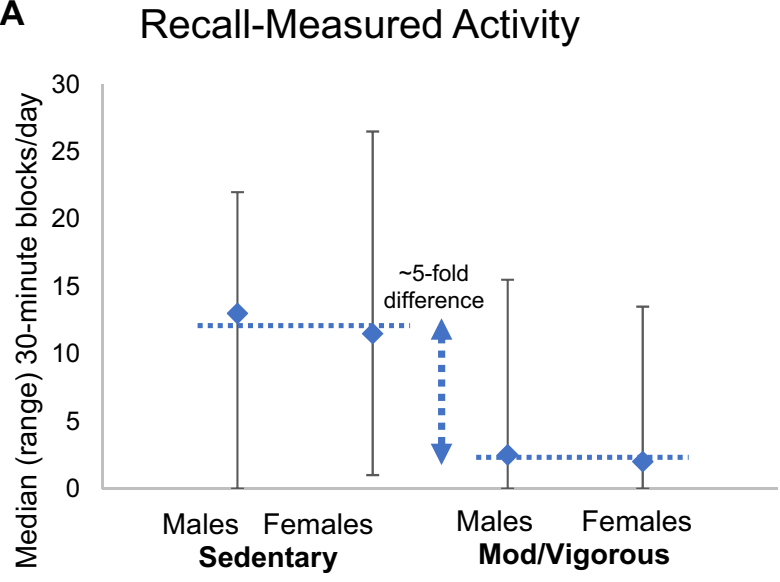

\section{B Accelerometer-Measured Activity}

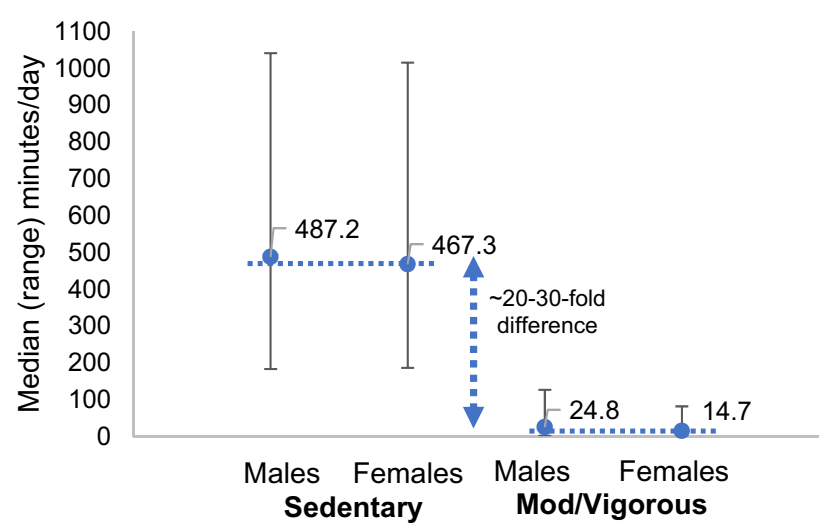

Fig. 1 Using a 3-day physical activity recall $\mathbf{A}$, as compared to accelerometer-measured activity $\mathbf{B}$, adolescents with overweight or obesity and type 2 diabetes (mean age 13.8 years, $60.6 \%$ female, $30.1 \%$ nonHispanic Black, $44.9 \%$ Hispanic) tended to overestimate time spent in moderate-vigorous physical activity and underestimate time spent sedentary. Although the units of comparison differ (30-min blocks for recall versus minutes/day for accelerometer), the discrepant ratios are evident: adolescents recalled spending approximately 5 times as much time sedentary as they spent in moderate-vigorous activity, while accelerometry revealed an approximately 20-30-fold greater time spent sedentary than in moderate-vigorous activity. This study highlights the notable contrast in perceived and actual activity and sedentary behavior among adolescents with overweight or obesity. Data from Rockette-Wagner et al. (24) dotted lines represent median time spent sedentary or in moderate-vigorous physical activity. Dashed arrow represents difference in medians. Bars represent range (recalled or accelerometer-measured) adolescents. Interestingly, in contrast to studies in adults, a study of 27 preschoolers by Byun et al. found that Fitbit Flex underestimated, rather than overestimated, moderateto-vigorous physical activity [28]. The validity of the wristbased Fitbit Charge HR (Fitbit, San Francisco CA) versus hip-based ActiGraph (ActiGraph LLC, Pensacola, FL) in 30 adolescents with congenital heart disease was evaluated by Voss et al. [29]. The authors found a strong association between Fitbit and ActiGraph for step count, and that Fitbit devices were worn approximately 30 min longer than accelerometers daily. Time spent in physical activity intensities was less well-correlated between the two devices, and between-device differences were greater in boys than girls. Similarly, in a study of 99 adolescents aged 10-17 in China, Yang et al. found that wristband-measured step count was highly correlated with ActiGraph step count, but that time spent on total physical activity and physical activity energy expenditure were less well correlated between devices [30•].

Poor compliance with wrist-worn activity trackers may limit the benefit of an mHealth intervention, as well as contribute to significant missing data. Strategies to encourage and remind adolescents to not only wear the device daily, but to charge and sync the device, should be built into mHealth interventions that use wrist-worn activity trackers. Alternate wristbands, with designs or extended sizes, may be available depending on the brand and device and may promote ongoing use. Smartwatches, which serve many other functions beyond activity tracking, may be more appealing to adolescents and may incentivize more long-term wear and thus data capture.

Activity-tracking apps, which may come pre-installed or be downloaded on smartphones, may offer another option for remote activity tracking. In healthy adults, Case et al. found that smartphone apps including Fitbit (Fitbit), Health Mate (Withings), and Moves (ProtoGeo Oy) accurately tracked step counts, while wearable devices including both waistband-worn and wrist-worn differed between each other to a greater degree [31]. To our knowledge, smartphone apps have not been validated for physical activity tracking in children or adolescents. However, given the ubiquity of smartphones and frequent use among adolescents, it is likely that these applications would capture the majority of an adolescent's sedentary behavior and light physical activity, although perhaps not more moderate or vigorous activities that would make carrying a smartphone difficult. Ultimately, to capture the greatest amount of data, as accurately as possible, a device must be easy to incorporate into one's daily routine, have a longlasting battery with effortless syncing, and be affordable. Smartwatches and phone-based trackers are promising in these regard but will require validation for use in children and adolescents. 


\section{Staying Engaged: Reminders, Encouragement, and Incentives}

\section{Text Messaging}

Even with the most accurate activity trackers, physical activity will not be accurately measured unless the participant remains engaged in the intervention and continues to wear the device as much as possible. The field of behavioral economics, which represents an intersection of concepts from psychology and economics, acknowledges that the human trait of bounded willpower sometimes leads to choices that are not optimal, despite knowing better [32]. Although a seemingly simple intervention, text messaging may be incorporated into mHealth physical activity interventions to help overcome this barrier of willpower by increasing the salience, or prominence, of a task, whether it is to engage in physical activity or simply to wear an activity tracker as requested for data capture. With current and emerging technology, text message reminders can be optimized to be highly salient to end-users: using geofencing technology, messages can be sent and tailored based on a user's location. This technology is currently being evaluated in the Location Initiated Individualized texts for African American Adolescent Health (LIITA3H) mobile application, in which culturally relevant messages are sent to users automatically when they are at a restaurant, with the aim of prompting healthy food choices (NCT03693144) [33]. Similar strategies that account for location or other inputs could be used to prompt physical activity; suggestions for exercise could be tailored by whether the user is at home or outside, near a park or a gym, or perhaps based on weather conditions. Alternatively, messages could be sent at individually tailored times during which an adolescent is most likely to be inactive or would be most receptive to a nudge to exercise. These times could be determined using ecological momentary assessment (EMA), in which repeated sampling (which may be done via app or text message) of a subject's current behaviors and experiences is collected in real time to minimize recall bias [34].

Text messages that provide feedback and encouragement may also help to overcome the barrier of bounded willpower. For example, feedback sent via text message to adults enrolled in a self-monitoring weight loss study led to significantly greater adherence and weight loss than those who did not receive feedback [35]. However, not all individuals respond positively to feedback, particularly if that feedback highlights a "failure" (e.g., to self-monitor, or to achieve a step count or weight loss goal) [36]. Assessment of participants' "behavioral phenotype" may allow text messages to be most appropriately tailored for ongoing engagement, minimizing the risk that individuals who tend to avoid bad news do not become avoidant and disengaged, while still supporting and providing feedback to those who are less discouraged by poor results or lack of progress. In all text messaging-based interventions, messages should be carefully constructed [37] and ideally co-designed with the target audience in order to avoid negative perceptions or disengagement [38, 39].

Because health behavior change interventions also tend to be more effective if guided by a behavioral theory [40], text message content should also be developed in a way to emphasize and reinforce concepts from such theories. Bandura's social cognitive theory [41], the most commonly used behavior change theory identified in a review of trials of wearable physical activity trackers for physical activity interventions targeting adolescents [42], posits that cognitive, environmental, and behavioral factors determine behavior. Text messages could be used to highlight or modify concepts within each of these factors: knowledge, expectations, and attitudes about exercise (cognitive factors); social norms about physical activity and access to safe places to exercise (environmental factors); and skill or comfort level as well as feeling of self-efficacy related to exercise (behavioral factors). Another health behavior change theory, the Theory of Planned Behavior (TPB) [43], is also commonly used for adolescent and adult health behavior change interventions [44]. According to the TPB, intention to engage in a behavior is predicted by 3 main constructs: attitude (belief about and evaluation of the consequences of the behavior), subjective norm (belief about what others want one to do and motivation to comply), and perceived behavioral control (belief about amount of control one has to successfully perform the behavior). Again, text messages could be designed to target constructs of the TPB, which should in turn impact intention and actual behavior.

Overall, as summarized in a systematic review by Ludwig et al., which included 13 studies reporting 11 interventions using text messaging to improve physical activity and sedentary behavior in adolescents 10-19 years of age, text messaging may be an effective method, but conclusions about the effectiveness are limited by the high heterogeneity of study design and outcome measures used [45•]. The authors note several important elements that could benefit future studies, including mixed-methods design, long-term followup, combination with a mobile app, and sending 3 or more text messages per week. In addition, two-way texting, which has been demonstrated to be acceptable by adolescents in a research setting, may be more engaging and effective than one-way [39]. Future research should also evaluate the costeffectiveness of text-message-based interventions in order to better understand the potential for scalability and implementation [46]. 


\section{Incentives and Gamification}

Strategic use of financial incentives can also promote engagement in mHealth interventions. In adults, financial incentives that leverage the behavioral economics concept of loss aversion [47-49] lead to greater engagement in physical activity. Loss aversion refers to the observation that losses tend to be felt more acutely than gains, or that strong negative feelings are often generated from the loss of something owned [47]. As shown by Patel et al., "loss-framing" of incentives, in which money was allocated to a virtual account and then removed for unmet step count goals, led to a significantly greater mean proportion of participant-days achieving a step count goal than the control group, while traditional "gain-framed" (money earned for step count goals met) and lottery-based incentives did not [48]. Loss-framed incentives have also been successfully shown to increase self-monitoring behaviors in adolescents with type 1 diabetes [49] but to our knowledge have not been evaluated as a strategy to promote physical activity in adolescents, with the exception of our current clinical trial (NCT04874415).

Incorporation of gameplay and game principles, either as elements of a larger non-game intervention (often referred to as "gamification") or as digital games designed specifically for health promotion, represents another method of encouraging engagement. Randomized clinical trials in adults have demonstrated the effectiveness of collaborative or cooperative efforts to achieve physical activity goals. For example, in the BE FIT trial, which included 200 adults comprising 94 families, participants randomized to earn points and progress through levels based on activity goal achievement attained step goals significantly more often than those randomized to the non-game control arm [50]. Evidence about the effectiveness of gamification on increasing physical activity in youth is more limited. In a longitudinal qualitative study of adolescents in Northern Ireland, Corepal et al. obtained feedback about a gamified intervention involving a pedometer-based step-count competition that also included rewards to encourage activity [51]. The authors found that competition motivated adolescents to initiate activity, but that negative feelings arose if they perceived only a remote chance of winning the competition or a prize. However, especially for girls, friends served as motivators to be more active. In a systematic review, Schwarz et al. identified features of digital health-promoting games that were associated with user engagement among youth; in addition to an attractive storyline, clear instructions that could be skipped, and a balance of educational and fun content, the inclusion of diverse characters that the user could identify with was highlighted as engaging [52]. As with text message content, game elements should also be adapted to the end-user to achieve the greatest acceptability and engagement.

\section{Remote Coaching? Telemedicine to Promote Physical Activity}

Telemedicine to provide pediatric weight management care has been studied for over a decade [53]. With the rapid uptake of telemedicine in March and April 2020 catalyzed by the COVID-19 pandemic, a much greater familiarity with telehealth has emerged [54], which may now support ongoing widespread use. Telemedicine appointments can overcome obstacles of travel, parking, missed school and work that can lead to high attrition rates in traditional clinics [55•]. In addition, telehealth-based programs allow more ready access to dieticians and specialty obesity clinics, particularly in rural areas [55•]. With high patient satisfaction and non-inferior clinical efficacy of telehealth as compared to in-person pediatric weight management programs [55•], such programs are highly promising alternative care models. For exercise programs, in particular, an initial in-person visit with a personal trainer [56] may be valuable to establish safe techniques and make follow-up visits conducted via telehealth more appropriate and effective.

Tele-exercise programs have been used successfully in children with cystic fibrosis [57], as well as in elderly adults, in which participation in a video conferencing-based supervised resistance exercise program resulted in increased muscle mass and lean soft tissue [58]. In youth-targeted interventions, telemedicine-based exercise coaching could also facilitate caregiver involvement, which has been associated with greater success in mHealth interventions [13]. In a randomized clinical trial of home-based "exergaming" (video games that require physical activity as part of game play), Staiano et al. paired telehealth coaching with parentsupported exergame play targeting 10-12-year-old youth

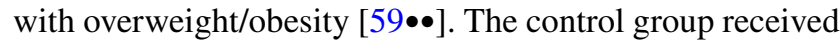
an exergame without coaching, while the intervention participants were assigned to participate in 3, 1-h gameplay sessions per week and videochat sessions with a fitness coach over 24 weeks. The intervention group experienced significantly greater rates of moderate to vigorous physical activity (measured by actigraphy) as well as improved blood pressure and total and LDL cholesterol.

In order to develop successful telemedicine programs with equity in mind, however, several potential barriers should be proactively addressed. Sharing their experience gained during the COVID-19 pandemic in New York City, New York, Woo Baidal et al. clearly delineate challenges to telehealth weight management programs designed for vulnerable children, including those of racial and ethnic minorities. These include language barriers, privacy concerns, reimbursement challenges, difficulty measuring effectiveness of programs remotely, and, importantly, the digital divide created by greater access to internet and smartphones among 
white, non-Hispanic families than Hispanic and black, nonHispanic families [60]. When targeting larger populations, the appropriateness of interventions must be considered for adolescents with chronic health conditions that require modifications to safely engage in physical activity. As with any intervention, the cost of the platform, including how it will be supported and maintained, must also be considered.

\section{Conclusions}

Mobile health technology continues to advance rapidly, and telehealth is now familiar to many health systems and patients. These facts lay the groundwork for exciting opportunities to bring physical activity interventions to patients, particularly digitally native adolescents, in a way that is potentially low cost and scalable. After first addressing issues of equity and access to digital technologies, one of the largest challenges that remains is how to initiate and sustain user engagement. Future studies of mHealth interventions targeting physical activity promotion in adolescents should clearly share engagement strategies and their effectiveness, such as wearable activity tracker compliance and patterns of missing data. For text message-based interventions, a description of how message content was developed should be shared, including health behavior change theory frameworks and end-user participation. Demonstration of effectiveness and comparative effectiveness of different financial incentive strategies is also needed for adolescent-targeted health behavior change interventions. In conclusion, ways to address and avoid potential pitfalls of mHealth and telehealthbased physical activity interventions for adolescents with obesity discussed in this review should allow intervention designers to capitalize on the promise of these interventions, maximizing effectiveness and scalability.

Funding ME Vajravelu is supported by NIH NIDDK grant K23DK125719.

\section{Declarations}

Conflict of Interest The authors have no competing interests to disclose.

Human and Animal Rights All reported studies/experiments with human or animal subjects performed by the authors have been previously published and complied with all applicable ethical standards (including the Helsinki declaration and its amendments, institutional/ national research committee standards, and international/national/institutional guidelines).

\section{References}

Papers of particular interest, published recently, have been highlighted as:
- Of importance

$\bullet$ Of major importance

1. Zhang X, Speakman JR. Genetic factors associated with human physical activity: are your genes too tight to prevent you exercising? Endocrinology. 2019;160(4):840-52.

2. Craigie AM, Lake AA, Kelly SA, Adamson AJ, Mathers JC. Tracking of obesity-related behaviours from childhood to adulthood: a systematic review. Maturitas. 2011;70(3):266-84.

3. Ward ZJ, Long MW, Resch SC, Giles CM, Cradock AL, Gortmaker SL. Simulation of growth trajectories of childhood obesity into adulthood. N Engl J Med. 2017;377(22):2145-53.

4. $\bullet$ US Department of Health and Human Services. Physical activity guidelines for Americans. Washington: DC, U.S; 2018. This comprehensive report describes evidence-based guidelines for physical activity in individuals of all ages, including adolescents.

5. Fedewa MV, Gist NH, Evans EM, Dishman RK. Exercise and insulin resistance in youth: a meta-analysis. Pediatrics. 2014;133(1):e163-74.

6. Ho M, Garnett SP, Baur LA, Burrows T, Stewart L, Neve M, et al. Impact of dietary and exercise interventions on weight change and metabolic outcomes in obese children and adolescents: a systematic review and meta-analysis of randomized trials. JAMA Pediatr. 2013;167(8):759-68.

7. Lee S, Deldin AR, White D, Kim Y, Libman I, Rivera-Vega M, et al. Aerobic exercise but not resistance exercise reduces intrahepatic lipid content and visceral fat and improves insulin sensitivity in obese adolescent girls: a randomized controlled trial. Am J Physiol Endocrinol Metab. 2013;305(10):E1222-9.

8. Pascoe M, Bailey AP, Craike M, Carter T, Patten R, Stepto $\mathrm{N}$, et al. Physical activity and exercise in youth mental health promotion: a scoping review. BMJ Open Sport Exerc Med. 2020;6(1):e000677.

9. Browne NT, Snethen JA, Greenberg CS, Frenn M, Kilanowski JF, Gance-Cleveland B, et al. When pandemics collide: the impact of COVID-19 on childhood obesity. J Pediatr Nurs. 2021;56:90-8.

10. Pew Research Center. Teens, Social Media \& Technology 2018.

11. Elvsaas IKO, Giske L, Fure B, Juvet LK. Multicomponent lifestyle interventions for treating overweight and obesity in children and adolescents: a systematic review and meta-analyses. $\mathbf{J}$ Obes. 2017;2017:5021902.

12. Zeller M, Kirk S, Claytor R, Khoury P, Grieme J, Santangelo M, et al. Predictors of attrition from a pediatric weight management program. J Pediatr. 2004;144(4):466-70.

13. Fedele DA, Cushing CC, Fritz A, Amaro CM, Ortega A. Mobile health interventions for improving health outcomes in youth: a meta-analysis. JAMA Pediatr. 2017;171(5):461-9.

14. Vandelanotte C, Muller AM, Short CE, Hingle M, Nathan N, Williams SL, et al. Past, present, and future of eHealth and mHealth research to improve physical activity and dietary behaviors. J Nutr Educ Behav. 2016;48(3):219-28 e1.

15. Lee S, Burns SF, White D, Kuk JL, Arslanian S. Effects of acute exercise on postprandial triglyceride response after a high-fat meal in overweight black and white adolescents. Int J Obes (Lond). 2013;37(7):966-71.

16. Lee S, Bacha F, Hannon T, Kuk JL, Boesch C, Arslanian S. Effects of aerobic versus resistance exercise without caloric restriction on abdominal fat, intrahepatic lipid, and insulin sensitivity in obese adolescent boys: a randomized, controlled trial. Diabetes. 2012;61(11):2787-95.

17. Lee S, Libman I, Hughan K, Kuk JL, Jeong JH, Zhang D, et al. Effects of exercise modality on insulin resistance and ectopic fat in adolescents with overweight and obesity: a randomized clinical trial. J Pediatr. 2019;206:91-8 e1. 
18. Yu WW, Lee S, Arslanian S, Tamim H, Kuk JL. Effects of exercise on resting metabolic rate in adolescents with overweight and obesity. Child Obes. 2021;17(4):249-56.

19. Organization WH. Global health risks: mortality and burden of disease attributable to selected major risks. 2009.

20. Young DR, Hivert MF, Alhassan S, Camhi SM, Ferguson JF, Katzmarzyk PT, et al. Sedentary behavior and cardiovascular morbidity and mortality: a science advisory from the American Heart Association. Circulation. 2016;134(13):e262-79.

21. Carson V, Hunter S, Kuzik N, Gray CE, Poitras VJ, Chaput JP, et al. Systematic review of sedentary behaviour and health indicators in school-aged children and youth: an update. Appl Physiol Nutr Metab. 2016;41(6 Suppl 3):S240-65.

22. Avery A, Anderson C, McCullough F. Associations between children's diet quality and watching television during meal or snack consumption: a systematic review. Matern Child Nutr. 2017;13(4)

23. Barnett TA, Kelly AS, Young DR, Perry CK, Pratt CA, Edwards $\mathrm{NM}$, et al. Sedentary Behaviors in today's youth: approaches to the prevention and management of childhood obesity: a scientific statement from the American Heart Association. Circulation. 2018;138(11):e142-59.

24. Rockette-Wagner B, Storti KL, Edelstein S, Delahanty LM, Galvin B, Jackson A, et al. Measuring physical activity and sedentary behavior in youth with type 2 diabetes. Child Obes. 2017;13(1):72-7.

25. Lockwood J, Jeffery A, Schwartz A, Manlhiot C, Schneiderman JE, McCrindle BW, et al. Comparison of a physical activity recall questionnaire with accelerometry in children and adolescents with obesity: a pilot study. Pediatr Obes. 2017;12(5):e41-5.

26. Henriksen A, Haugen Mikalsen M, Woldaregay AZ, Muzny M, Hartvigsen G, Hopstock LA, et al. Using fit fitness trackers and smartwatches to measure physical activity in research: analysis of consumer wrist-worn wearables. J Med Internet Res. 2018;20(3):e110. (This study examines the availability of wrist-worn fitness wearables and highlights the constantly changing landscape of devices and brands. Fitbit was the most most commonly used brand in studies registered on ClinicalTrials.gov).

27. Feehan LM, Geldman J, Sayre EC, Park C, Ezzat AM, Yoo JY, et al. Accuracy of fitbit devices: systematic review and narrative syntheses of quantitative data. JMIR Mhealth Uhealth. 2018;6(8):e10527.

28. Byun W, Kim Y, Brusseau TA. The use of a fitbit device for assessing physical activity and sedentary behavior in preschoolerS. J Pediatr. 2018;199:35-40.

29. Voss C, Gardner RF, Dean PH, Harris KC. Validity of commercial activity trackers in children with congenital heart disease. Can J Cardiol. 2017;33(6):799-805.

30. Yang X, Jago R, Zhang Q, Wang YY, Zhang J, Zhao WH. Validity and reliability of the wristband activity monitor in free-living children aged 10-17 yearS. Biomed Environ Sci. 2019;32(11):812-22. This prospective study determined that a wristband activity monitor was reliable and valid for measuring physical activity in youth ages 10-17 years as compared to a research-grade accelerometer, but found that intensity of activity impacted correlations.

31. Case MA, Burwick HA, Volpp KG, Patel MS. Accuracy of smartphone applications and wearable devices for tracking physical activity data. JAMA. 2015;313(6):625-6.

32. Mullainathan S, Thaler RH. Behavioral Economics. 2000.

33. Woolford SJ, Moon J. Development of the Location Initiated Individualized Texts for African American Adolescent Health (Liita3h) mobile application. J Adolesc Health. 2016;58(2).

34. Shiffman S, Stone AA, Hufford MR. Ecological momentary assessment. Annu Rev Clin Psychol. 2008;4:1-32.
35. Burke LE, Conroy MB, Sereika SM, Elci OU, Styn MA, Acharya $\mathrm{SD}$, et al. The effect of electronic self-monitoring on weight loss and dietary intake: a randomized behavioral weight loss trial. Obesity (Silver Spring). 2011;19(2):338-44.

36. Kangovi S, Asch DA. Behavioral phenotyping in health promotion: embracing or avoiding failure. JAMA. 2018;319(20):2075-6.

37. Woolford SJ, Barr KL, Derry HA, Jepson CM, Clark SJ, Strecher VJ, et al. OMG do not say LOL: obese adolescents' perspectives on the content of text messages to enhance weight loss efforts. Obesity (Silver Spring). 2011;19(12):2382-7.

38. Smith KL, Kerr DA, Fenner AA, Straker LM. Adolescents just do not know what they want: a qualitative study to describe obese adolescents' experiences of text messaging to support behavior change maintenance post intervention. J Med Internet Res. 2014;16(4):e103.

39. Partridge SR, Raeside R, Singleton A, Hyun K, Redfern J. Effectiveness of text message interventions for weight management in adolescents: systematic review. JMIR Mhealth Uhealth. 2020;8(5):e15849.

40. Webb TL, Joseph J, Yardley L, Michie S. Using the internet to promote health behavior change: a systematic review and meta-analysis of the impact of theoretical basis, use of behavior change techniques, and mode of delivery on efficacy. J Med Internet Res. 2010;12(1):e4.

41. Bandura A. Social foundations of thought and action: a social cognitive theory. Upper Saddle River, NJ: Prentice Hall; 1986.

42. Bohm B, Karwiese SD, Bohm H, Oberhoffer R. Effects of mobile health including wearable activity trackers to increase physical activity outcomes among healthy children and adolescents: systematic review. JMIR Mhealth Uhealth. 2019;7(4):e8298.

43. Azjen I. The Theory of Planned Behavior. Organ Behav Hum Decis Process. 1991;50:179-211.

44. Muzaffar H, Chapman-Novakofski K, Castelli DM, Scherer JA. The HOT (Healthy Outcome for Teens) project. Using a webbased medium to influence attitude, subjective norm, perceived behavioral control and intention for obesity and type 2 diabetes prevention. Appetite. 2014;72:82-9.

45. Ludwig K, Arthur R, Sculthorpe N, Fountain H, Buchan DS. Text messaging interventions for improvement in physical activity and sedentary behavior in youth: systematic review. JMIR Mhealth Uhealth. 2018;6(9):e10799. This systematic review of text messaging interventions for improvement in physical activity and sedentary behavior in youth highlights the heterogeneity of previous studies and identifies several areas for improvement for future interventions.

46. Tully L, Burls A, Sorensen J, El-Moslemany R, O'Malley G. Mobile health for pediatric weight management: systematic scoping review. JMIR Mhealth Uhealth. 2020;8(6):e16214.

47. Kahneman D, Tversky A. Prospect theory: an analysis of decision under risk. Econometrics. 1979;47(2):263-92.

48. Patel MS, Asch DA, Rosin R, Small DS, Bellamy SL, Heuer J, et al. Framing financial incentives to increase physical activity among overweight and obese adults: a randomized, controlled trial. Ann Intern Med. 2016;164(6):385-94.

49. Wong CA, Miller VA, Murphy K, Small D, Ford CA, Willi SM, et al. Effect of financial incentives on glucose monitoring adherence and glycemic control among adolescents and young adults with type 1 diabetes: a randomized clinical trial. JAMA Pediatr. 2017;171(12):1176-83.

50. Patel MS, Benjamin EJ, Volpp KG, Fox CS, Small DS, Massaro JM, et al. Effect of a game-based intervention designed to enhance social incentives to increase physical activity among families: the BE FIT Randomized Clinical Trial. JAMA Intern Med. 2017;177(11):1586-93. 
51. Corepal R, Best P, O'Neill R, Tully MA, Edwards M, Jago R, et al. Exploring the use of a gamified intervention for encouraging physical activity in adolescents: a qualitative longitudinal study in Northern Ireland. BMJ Open. 2018;8(4):e019663.

52. Schwarz AF, Huertas-Delgado FJ, Cardon G, DeSmet A. Design features associated with user engagement in digital games for healthy lifestyle promotion in youth: a systematic review of qualitative and quantitative studies. Games Health J. 2020;9(3):150-63.

53. Cohen GM, Irby MB, Boles K, Jordan C, Skelton JA. Telemedicine and pediatric obesity treatment: review of the literature and lessons learned. Clin Obes. 2012;2(3-4):103-11.

54. Schinasi DA, Foster CC, Bohling MK, Barrera L, Macy ML. Attitudes and perceptions of telemedicine in response to the COVID-19 pandemic: a survey of naive healthcare providers. Front Pediatr. 2021;9:647937.

55. DeSilva S, Vaidya SS. The Application of telemedicine to pediatric obesity: lessons from the past decade. Telemed J E Health. 2021;27(2):159-66. This review examined studies of telemedicine for pediatric weight management from the past decade. The authors found that telemedicine studies demonstrated noninferiority by weight status improvement, that attrition rates were not significantly different between telemedicine and in-person cohorts, and that patient satisfaction with telemedicine was high.

56. O'Hara VM, Johnston SV, Browne NT. The paediatric weight management office visit via telemedicine: pre- to postCOVID-19 pandemic. Pediatr Obes. 2020;15(8):e12694.
57. Chen JJ, Cooper DM, Haddad F, Sladkey A, Nussbaum E, Radom-Aizik S. Tele-exercise as a promising tool to promote exercise in children with cystic fibrosis. Front Public Health. 2018;6:269.

58. Hong J, Kim J, Kim SW, Kong HJ. Effects of home-based teleexercise on sarcopenia among community-dwelling elderly adults: Body composition and functional fitness. Exp Gerontol. 2017;87(Pt A):33-9.

59.• Staiano AE, Beyl RA, Guan W, Hendrick CA, Hsia DS, Newton RL Jr. Home-based exergaming among children with overweight and obesity: a randomized clinical trial. Pediatr Obes. 2018;13(11):724 33. This prospective randomized clinical trial demonstrated the efficacy of a home-based exergaming intervention targeting children with overweight and obesity. Important aspects of this intervention include remote coaching via videochat and the ability to participate in an engaging physical activity at home with parental support.

60. Woo Baidal JA, Chang J, Hulse E, Turetsky R, Parkinson K, Rausch JC. Zooming toward a telehealth solution for vulnerable children with obesity during coronavirus disease 2019 . Obesity (Silver Spring). 2020;28(7):1184-6.

Publisher's Note Springer Nature remains neutral with regard to jurisdictional claims in published maps and institutional affiliations. 